\title{
On AP-Henstock Integrals of Interval-Valued Functions and Fuzzy-Number-Valued Functions
}

\author{
Muawya Elsheikh Hamid ${ }^{1 *}$, Alshaikh Hamed Elmuiz ${ }^{1}$, Mohammed Eldirdiri Sheima ${ }^{2}$ \\ ${ }^{1}$ School of Management, Ahfad University for Women, Omdurman, Sudan \\ ${ }^{2}$ Faculty of Engineering, University of Khartoum, Khartoum, Sudan \\ Email: ^mowia-84@hotmail.com, ^muawya.ebrahim@gmail.com, almoizalsheikh1@windowslive.com, sheima77@gmail.com
}

How to cite this paper: Hamid, M.E., Elmuiz, A.H. and Sheima, M.E. (2016) On AP-Henstock Integrals of Interval-Valued Functions and Fuzzy-Number-Valued Functions. Applied Mathematics, 7, 2285-2295. http://dx.doi.org/10.4236/am.2016.718180

Received: October 8, 2016

Accepted: November 29, 2016

Published: December 2, 2016

Copyright $\odot 2016$ by authors and Scientific Research Publishing Inc. This work is licensed under the Creative Commons Attribution International License (CC BY 4.0). http://creativecommons.org/licenses/by/4.0/

\begin{abstract}
In 2000, Wu and Gong [1] introduced the thought of the Henstock integrals of interval-valued functions and fuzzy-number-valued functions and obtained a number of their properties. The aim of this paper is to introduce the thought of the APHenstock integrals of interval-valued functions and fuzzy-number-valued functions which are extensions of [1] and investigate a number of their properties.
\end{abstract}

\section{Keywords}

Fuzzy Numbers, AP-Henstock Integrals of Interval-Valued Functions, AP-Henstock Integrals of Fuzzy-Number-Valued Functions

\section{Introduction}

As it is well known, the Henstock integral for a real function was first defined by Henstock [2] in 1963. The Henstock integral is a lot of powerful and easier than the Lebesgue, Wiener and Richard Phillips Feynman integrals. Furthermore, it is also equal to the Denjoy and the Perron integrals [2] [3]. In 2016, Hamid and Elmuiz [4] introduced the concept of the Henstock-Stieltjes (HS) integrals of interval-valued functions and fuzzy-number-valued functions and discussed a number of their properties.

In this paper, we introduce the concept of the AP-Henstock integrals of interval-valued functions and fuzzy-number-valued functions and discuss some of their properties.

The paper is organized as follows. In Section 2, we have a tendency to provide the preliminary terminology used in this paper. Section 3 is dedicated to discussing the AP-Henstock integral of interval-valued functions. In Section 4, we introduce the APHenstock integral of fuzzy-number-valued functions. The last section provides conclusions. 


\section{Preliminaries}

Let $E$ be a measurable set and let $C$ be a real number. The density of $E$ at $C$ is defined by

$$
d_{c} E=\lim _{h \rightarrow 0^{+}} \frac{\mu(E \bigcap(c-h, c+h))}{2 h},
$$

provided the limit exists. The point $c$ is called a point of density of $E$ if $d_{c} E=1$. The set $E^{d}$ represents the set of all points $x \in E$ such that $x$ is a point of density of E.

A measurable set $S_{x} \subseteq[a, b]$ is called an approximate neighborhood (br.ap-nbd) of $x \in[a, b]$ if it containing $x$ as a point of density. We choose an ap-nbd $S_{x} \subseteq[a, b]$ for each $x \in E \subseteq[a, b]$ and denote a choice on $E$ by $S=\left\{S_{x}: x \in E\right\}$. A tagged interval-point pair $([u, v], \xi)$ is said to be $S$-fine if $\xi \in[u, v]$ and $u, v \in S_{\xi}$.

A division $P$ is a finite collection of interval-point pairs $\left\{\left(\left[u_{i}, v_{i}\right] ; \xi_{i}\right)\right\}_{i=1}^{n}$, where $\left\{\left[u_{i}, v_{i}\right]\right\}_{i=1}^{n}$ are non-overlapping subintervals of $[a, b]$. We say that $P=\left\{\left(\left[u_{i}, v_{i}\right] ; \xi_{i}\right)\right\}_{i=1}^{n}$ is

1) a division of $[a, b]$ if $\bigcup_{i=1}^{n}\left[u_{i}, v_{i}\right]=[a, b]$;

2) $S$-fine division of $[a, b]$ if $\xi_{i} \in\left[u_{i}, v_{i}\right]$ and $\left(\left[u_{i}, v_{i}\right], \xi_{i}\right)$ is $S$-fine for all $i=1,2, \cdots, n$.

Definition 2.1. [2] [3] A real-valued function $f:[a, b] \rightarrow R$ is said to be Henstock integrable to $A$ on $[a, b]$ if for every $\varepsilon>0$, there is a function $\delta(t)>0$ such that for any $\delta$-fine division $P=\left\{\left[u_{i}, v_{i}\right] ; \xi_{i}\right\}_{i=1}^{n}$ of $[a, b]$, we have

$$
\left|\sum_{i=1}^{n} f\left(\xi_{i}\right)\left(v_{i}-u_{i}\right)-A\right|<\varepsilon
$$

where the sum $\sum$ is understood to be over $P$ and we write $(H) \int_{a}^{b} f(t) \mathrm{d} t=A$, and $f \in H[a, b]$.

Definition 2.2. [5] A function $f:[a, b] \rightarrow R$ is AP-Henstock integrable if there exists a real number $A \in R$ such that for each $\varepsilon>0$ there is a choice $S$ such that

$$
\left|\sum_{i=1}^{n} f\left(\xi_{i}\right)\left(v_{i}-u_{i}\right)-A\right|<\varepsilon
$$

for each $S$-fine division $P=\left\{\left(\left[u_{i}, v_{i}\right] ; \xi_{i}\right)\right\}_{i=1}^{n}$ of $[a, b]$. A is called AP-Henstock integral of $f$ on $[a, b]$, and we write $A=(A P H) \int_{a}^{b} f$.

Theorem 2.1. If $f$ and $g$ are AP-Henstock integrable on $[a, b]$ and $f \leq g$ almost everywhere on $[a, b]$, then

$$
(A P H) \int_{a}^{b} f \leq(A P H) \int_{a}^{b} g .
$$


Proof. The proof is similar to the Theorem 3.6 in [3].

\section{The AP-Henstock Integral of Interval-Valued Functions}

In this section, we shall give the definition of the AP-Henstock integrals of interval-valued functions and discuss some of their properties.

Definition 3.1. [1] Let

$I_{R}=\left\{I=\left[I^{-}, I^{+}\right]: \mathrm{I}\right.$ is the closed bounded interval on the real line $\left.R\right\}$.

For $A, B \in I_{R}$, we define $A \leq B$ iff $A^{-} \leq B^{-}$and $A^{+} \leq B^{+}, A+B=C$ iff $C^{-}=A^{-}+B^{-}$and $C^{+}=A^{+}+B^{+}$, and $A \cdot B=\{a \cdot b: a \in A, b \in B\}$, where

$$
(A \cdot B)^{-}=\min \left\{A^{-} \cdot B^{-}, A^{-} \cdot B^{+}, A^{+} \cdot B^{-}, A^{+} \cdot B^{+}\right\}
$$

and

$$
(A \cdot B)^{+}=\max \left\{A^{-} \cdot B^{-}, A^{-} \cdot B^{+}, A^{+} \cdot B^{-}, A^{+} \cdot B^{+}\right\} .
$$

Define $\mathrm{d}(A, B)=\max \left(\left|A^{-}-B^{-}\right|,\left|A^{+}-B^{+}\right|\right)$as the distance between intervals $A$ and $B$.

Definition 3.2. [1] Let $F:[a, b] \rightarrow I_{R}$ be an interval-valued function. $I_{0} \in I_{R}$, for every $\varepsilon>0$ there is a $\delta(t)>0$ such that for any $\delta$-fine division $P=\left\{\left[u_{i}, v_{i}\right], \xi_{i}\right\}_{i=1}^{n}$ we have

$$
\mathrm{d}\left(\sum_{i=1}^{n} F\left(\xi_{i}\right)\left(v_{i}-u_{i}\right), I_{0}\right)<\varepsilon,
$$

then $F(t)$ is said to be Henstock integrable over $[a, b]$ and write $(I H) \int_{a}^{b} F(t) \mathrm{d} t=I_{0}$. For brevity, we write $F(t) \in I H[a, b]$.

Definition 3.3. A interval-valued function $F:[a, b] \rightarrow I_{R}$ is AP-Henstock integrable to $I_{0} \in I_{R}$, if for every $\varepsilon>0$ there exists a choice $S$ on $[a, b]$ such that

$$
\mathrm{d}\left(\sum_{i=1}^{n} F\left(\xi_{i}\right)\left(v_{i}-u_{i}\right), I_{0}\right)<\varepsilon
$$

whenever $P=\left\{\left(\left[u_{i}, v_{i}\right] ; \xi_{i}\right)\right\}_{i=1}^{n}$ is a $S$-fine division of $[a, b]$, we write $(A P I H) \int_{a}^{b} F=I_{0}$ and $F \in A P I H[a, b]$.

Theorem 3.1. If $\mathrm{F} \in \mathrm{APIH}[a, b]$, then the integral value is unique.

Proof. Let integral value is not unique and let $B_{1}=(A P I H) \int_{a}^{b} F$ and $B_{2}=(A P I H) \int_{a}^{b} F$. Let $\varepsilon>0$ be given. Then there exists a choice $S$ on $[a, b]$ such that

$$
\mathrm{d}\left(\sum_{i=1}^{n} F\left(\xi_{i}\right)\left(v_{i}-u_{i}\right), B_{1}\right)<\frac{\varepsilon}{2}
$$




$$
\mathrm{d}\left(\sum_{i=1}^{n} F\left(\xi_{i}\right)\left(v_{i}-u_{i}\right), B_{2}\right)<\frac{\varepsilon}{2}
$$

whenever $P=\left\{\left(\left[u_{i}, v_{i}\right] ; \xi_{i}\right)\right\}_{i=1}^{n}$ is a $S$-fine division of $[a, b]$.

Whence it follows from the Triangle Inequality that:

$$
\mathrm{d}\left(B_{1}, B_{2}\right)=\mathrm{d}\left(\sum_{i=1}^{n} F\left(\xi_{i}\right)\left(v_{i}-u_{i}\right), B_{1}\right)+\mathrm{d}\left(\sum_{i=1}^{n} F\left(\xi_{i}\right)\left(v_{i}-u_{i}\right), B_{2}\right)<\frac{\varepsilon}{2}+\frac{\varepsilon}{2}=\varepsilon .
$$

Since for $\forall \varepsilon>0$, there exists a choice $S$ on $[a, b]$ as above so $B_{1}=B_{2}$.

Theorem 3.2. An interval-valued function $F \in A P I H[a, b]$ if and only if $F^{-}, F^{+} \in A P H[a, b]$ and

$$
(A P I H) \int_{a}^{b} F=\left[(A P H) \int_{a}^{b} F^{-},(A P H) \int_{a}^{b} F^{+}\right] .
$$

Proof. Let $F \in A P I H[a, b]$, from Definition 3.3 there is a unique interval number $I_{0}=\left[I_{0}^{-}, I_{0}^{+}\right]$with the property that for any $\varepsilon>0$ there exists a choice $S$ on $[a, b]$ such that

$$
\mathrm{d}\left(\sum_{i=1}^{n} F\left(\xi_{i}\right)\left(v_{i}-u_{i}\right), I_{0}\right)<\varepsilon
$$

whenever $P=\left\{\left(\left[u_{i}, v_{i}\right] ; \xi_{i}\right)\right\}_{i=1}^{n}$ is a $S$-fine division of $[a, b]$. Since $v_{i}-u_{i} \geq 0$ for $1 \leq i \leq n$, we have

$$
\begin{aligned}
& \mathrm{d}\left(\sum_{i=1}^{n} F\left(\xi_{i}\right)\left(v_{i}-u_{i}\right), I_{0}\right)<\varepsilon \\
& =\max \left(\left|\left[\sum_{i=1}^{n} F\left(\xi_{i}\right)\left(v_{i}-u_{i}\right)\right]^{-}-I_{0}^{-}\right|,\left|\left[\sum_{i=1}^{n} F\left(\xi_{i}\right)\left(v_{i}-u_{i}\right)\right]^{+}-I_{0}^{+}\right|\right)<\varepsilon . \\
& =\max \left(\left|\sum_{i=1}^{n} F^{-}\left(\xi_{i}\right)\left(v_{i}-u_{i}\right)-I_{0}^{-}\right|,\left|\sum_{i=1}^{n} F^{+}\left(\xi_{i}\right)\left(v_{i}-u_{i}\right)-I_{0}^{+}\right| \mid\right)<\varepsilon .
\end{aligned}
$$

Hence $\left|\sum_{i=1}^{n} F^{-}\left(\xi_{i}\right)\left(v_{i}-u_{i}\right)-I_{0}^{-}\right|<\varepsilon, \quad\left|\sum_{i=1}^{n} F^{+}\left(\xi_{i}\right)\left(v_{i}-u_{i}\right)-I_{0}^{+}\right|<\varepsilon$ whenever $P=\left\{\left(\left[u_{i}, v_{i}\right] ; \xi_{i}\right)\right\}_{i=1}^{n}$ is a $S$-fine division of $[a, b]$. Thus $F^{-}, F^{+} \in A P H[a, b]$ and

$$
(A P I H) \int_{a}^{b} F=\left[(A P H) \int_{a}^{b} F^{-},(A P H) \int_{a}^{b} F^{+}\right] .
$$

Conversely, let $F^{-}, F^{+} \in A P H[a, b]$. Then there exists $H_{1}, H_{2} \in R$ with the property that given $\varepsilon>0$ there exists a choice $S$ on $[a, b]$ such that

$$
\left|\sum_{i=1}^{n} F^{-}\left(\xi_{i}\right)\left(v_{i}-u_{i}\right)-H_{1}\right|<\varepsilon,\left|\sum_{i=1}^{n} F^{+}\left(\xi_{i}\right)\left(v_{i}-u_{i}\right)-H_{2}\right|<\varepsilon
$$

whenever $P=\left\{\left(\left[u_{i}, v_{i}\right] ; \xi_{i}\right)\right\}_{i=1}^{n}$ is a $S$-fine division of $[a, b]$. We define $I_{0}=\left[H_{1}, H_{2}\right]$, then if $P=\left\{\left[u_{i}, v_{i}\right], \xi_{i}\right\}_{i=1}^{n}$ is a $S$-fine division of $[a, b]$, we have 


$$
\mathrm{d}\left(\sum_{i=1}^{n} F\left(\xi_{i}\right)\left(v_{i}-u_{i}\right), I_{0}\right)<\varepsilon
$$

Hence $F:[a, b] \rightarrow I_{R}$ is AP-Henstock integrable on $[a, b]$.

Theorem 3.3. If $F, G \in A P I H[a, b]$ and $\beta, \gamma \in R$. Then $\beta F+\gamma G \in A P I H[a, b]$ and

$$
(A P I H) \int_{a}^{b}(\beta F+\gamma G)=\beta(A P I H) \int_{a}^{b} F+\gamma(A P I H) \int_{a}^{b} G .
$$

Proof. If $F, G \in A P I H[a, b]$, then $F^{-}, F^{+}, G^{-}, G^{+} \in A P H[a, b]$ by Theorem 3.2. Hence $\beta F^{-}+\gamma G^{-}, \beta F^{-}+\gamma G^{+}, \beta F^{+}+\gamma G^{-}, \beta F^{+}+\gamma G^{+} \in A P H[a, b]$.

(1) If $\beta>0$ and $\gamma>0$, then

$$
\begin{aligned}
(\text { APH }) \int_{a}^{b}(\beta F+\gamma G)^{-} & =(\text {APH }) \int_{a}^{b}\left(\beta F^{-}+\gamma G^{-}\right) \\
& =\beta(\text { APH }) \int_{a}^{b} F^{-}+\gamma(\text { APH }) \int_{a}^{b} G^{-} \\
& =\beta\left((\text { APIH }) \int_{a}^{b} F\right)^{-}+\gamma\left((\text { APIH }) \int_{a}^{b} G\right)^{-} \\
& =\left(\beta(\text { APIH }) \int_{a}^{b} F+\gamma(\text { APIH }) \int_{a}^{b} G\right)^{-}
\end{aligned}
$$

(2) If $\beta<0$ and $\gamma<0$, then

$$
\begin{aligned}
(\text { APH }) \int_{a}^{b}(\beta F+\gamma G)^{-} & =(A P H) \int_{a}^{b}\left(\beta F^{+}+\gamma G^{+}\right) \\
& =\beta(A P H) \int_{a}^{b} F^{+}+\gamma(A P H) \int_{a}^{b} G^{+} \\
& =\beta\left((A P I H) \int_{a}^{b} F\right)^{+}+\gamma\left((A P I H) \int_{a}^{b} G\right)^{+} \\
& =\left(\beta(\text { APIH }) \int_{a}^{b} F+\gamma(\text { APIH }) \int_{a}^{b} G\right)^{-}
\end{aligned}
$$

(3) If $\beta>0$ and $\gamma<0$, (or $\beta<0$ and $\gamma<0$, ), then

$$
\begin{aligned}
(\text { APH }) \int_{a}^{b}(\beta F+\gamma G)^{-} & =(A P H) \int_{a}^{b}\left(\beta F^{-}+\gamma G^{+}\right) \\
& =\beta(\text { APH }) \int_{a}^{b} F^{-}+\gamma(A P H) \int_{a}^{b} G^{+} \\
& =\beta\left(((\text { APIH })) \int_{a}^{b} F\right)^{-}+\gamma\left((\text { APIH }) \int_{a}^{b} G\right)^{+} \\
& =\left(\beta(\text { APIH }) \int_{a}^{b} F+\gamma(\text { APIH }) \int_{a}^{b} G\right)^{-}
\end{aligned}
$$

Similarly, for four cases above we have 


$$
(A P H) \int_{a}^{b}(\beta F+\gamma G)^{+}=\left(\beta(A P I H) \int_{a}^{b} F+\gamma(A P I H) \int_{a}^{b} G\right)^{+} .
$$

Hence by Theorem $3.2 \beta F+\gamma G \in A P I H[a, b]$ and

$$
(A P I H) \int_{a}^{b}(\beta F+\gamma G)=\beta(A P I H) \int_{a}^{b} F+\gamma(A P I H) \int_{a}^{b} G .
$$

Theorem 3.4. If $F \in A P I H[a, c]$ and $F \in A P I H[c, b]$, then $F \in A P I H[a, b]$ and

$$
(A P I H) \int_{a}^{b} F=(A P I H) \int_{a}^{c} F+(A P I H) \int_{c}^{b} F
$$

Proof. If $F \in A P I H[a, c]$ and $F \in A P I H[c, b]$, then by Theorem 3.2

$F^{-}, F^{+} \in A P H[a, c]$ and $F^{-}, F^{+} \in A P H[c, b]$. Hence $F^{-}, F^{+} \in A P H[a, b]$ and

$$
\begin{aligned}
(A P H) \int_{a}^{b} F^{-} & =(A P H) \int_{a}^{c} F^{-}+(A P H) \int_{c}^{b} F^{-} \\
& =\left((A P I H) \int_{a}^{c} F+(A P I H) \int_{c}^{b} F\right)^{-} .
\end{aligned}
$$

Similarly, $(A P H) \int_{a}^{b} F^{+}=\left((A P I H) \int_{a}^{c} F+(A P I H) \int_{c}^{b} F\right)^{+}$. Hence by Theorem 3.2 $F \in A P I H[a, b]$ and

$$
(A P I H) \int_{a}^{b} F=(A P I H) \int_{a}^{c} F+(A P I H) \int_{c}^{b} F
$$

Theorem 3.5. If $F \leq G$ nearly everywhere on $[a, b]$ and $F, G \in A P I H[a, b]$, then

$$
(A P I H) \int_{a}^{b} F \leq(A P I H) \int_{a}^{b} G
$$

Proof. Let $F \leq G$ nearly everywhere on $[a, b]$ and $F, G \in A P I H[a, b]$ Then $F^{-}, F^{+}, G^{-}, G^{+} \in A P H[a, b]$ and $F^{-} \leq G^{-}, F^{+} \leq G^{+}$nearly everywhere on $[a, b]$ By Theorem $2.1(A P H) \int_{a}^{b} F^{-} \leq(A P H) \int_{a}^{b} G^{-}$and $(A P H) \int_{a}^{b} F^{+} \leq(A P H) \int_{a}^{b} G^{+}$. Hence

$$
(A P I H) \int_{a}^{b} F \leq(A P I H) \int_{a}^{b} G
$$

by Theorem 3.2.

Theorem 3.6. Let $F, G \in A P I H[a, b]$ and $\mathrm{d}(F, G)$ is Lebesgue integrable on $[a, b]$. Then

$$
\mathrm{d}\left((A P I H) \int_{a}^{b} F,(A P I H) \int_{a}^{b} G\right) \leq(L) \int_{a}^{b} \mathrm{~d}(F, G) .
$$

Proof. By definition of distance, 


$$
\begin{aligned}
& \mathrm{d}\left((\text { APIH }) \int_{a}^{b} F,(\text { APIH }) \int_{a}^{b} G\right) \\
& =\max \left(\left|\left((A P I H) \int_{a}^{b} F\right)^{-}-\left((A P I H) \int_{a}^{b} G\right)^{-}\right|,\left|\left((A P I H) \int_{a}^{b} F\right)^{+}-\left((A P I H) \int_{a}^{b} G\right)^{+}\right|\right) \\
& =\max \left(\left|(A P H) \int_{a}^{b}\left(F^{-}-G^{-}\right)\right|,(A P H) \int_{a}^{b}\left(F^{+}-G^{+}\right) \mid\right) \\
& \leq \max \left((L) \int_{a}^{b}\left|F^{-}-G^{-}\right|,(L) \int_{a}^{b}\left|F^{+}-G^{+}\right|\right) \\
& \leq(L) \int_{a}^{b} \max \left(\left|F^{-}-G^{-}\right|,\left|F^{+}-G^{+}\right|\right) \\
& =(L) \int_{a}^{b} \mathrm{~d}(F, G) .
\end{aligned}
$$

\section{The AP-Henstock Integral of Fuzzy-Number-Valued Functions}

This section introduces the concept of the AP-Henstock integral of fuzzy-numbervalued functions and investigates some of their properties.

Definition 4.1. [6] [7] [8] Let $\tilde{A} \in F(R)$ be a fuzzy subset on $R$. If for any $\lambda \in[0,1], \quad A_{\lambda}=\left[A_{\lambda}^{-}, A_{\lambda}^{+}\right]$and $A_{1} \neq \phi$, where $A_{\lambda}=\{t: \tilde{A}(t) \geq \lambda\}$, then $\tilde{A}$ is called a fuzzy number. If $\tilde{A}$ is convex, normal, upper semi-continuous and has the compact support, we say that $\tilde{A}$ is a compact fuzzy number.

Let $\tilde{R}$ denote the set of all fuzzy numbers.

Definition 4.2. [6] Let $\tilde{A}, \tilde{B} \in \tilde{R}$, we define $\tilde{A} \leq \tilde{B}$ iff $A_{\lambda} \leq B_{\lambda}$ for all $\lambda \in(0,1]$, $\tilde{A}+\tilde{B}=\tilde{C} \quad$ iff $A_{\lambda}+B_{\lambda}=C_{\lambda}$ for any $\lambda \in(0,1], \quad \tilde{A} \cdot \tilde{B}=\tilde{D}$ iff $A_{\lambda} \cdot B_{\lambda}=D_{\lambda}$ for any $\lambda \in(0,1]$.

For $\tilde{A}, \tilde{B} \in \tilde{R}^{C}, \quad D(\tilde{A}, \tilde{B})=\sup _{\lambda \in[0,1]} \mathrm{d}\left(A_{\lambda}, B_{\lambda}\right)$ is called the distance between $\tilde{A}$ and $\tilde{B}$.

Lemma 4.1. [9] If a mapping $H:[0,1] \rightarrow I_{R}, \quad \lambda \rightarrow H(\lambda)=\left[m_{\lambda}, n_{\lambda}\right]$, satisfies $\left[m_{\lambda_{1}}, n_{\lambda_{1}}\right] \supset\left[m_{\lambda_{2}}, n_{\lambda_{2}}\right]$ when $\lambda_{1}<\lambda_{2}$, then

$$
\tilde{A}:=\bigcup_{\lambda \in(0,1]} \lambda H(\lambda) \in \tilde{R}
$$

and

$$
A_{\lambda}=\bigcap_{n=1}^{\infty} H\left(\lambda_{n}\right)
$$

where $\lambda_{n}=\left[1-\frac{1}{(n+1)}\right] \lambda$.

Definition 4.3. [1] Let $\tilde{F}:[a, b] \rightarrow \tilde{R}$. If the interval-valued function $F_{\lambda}(t)=\left[F_{\lambda}^{-}(t), F_{\lambda}^{+}(t)\right]$ is Henstock integrable on $[a, b]$ for any $\lambda \in(0,1]$, then we say that $\tilde{F}(t)$ is Henstock integrable on $[a, b]$ and the integral value is defined by 


$$
\begin{aligned}
(F H) \int_{a}^{b} \tilde{F}(t) \mathrm{d} t & :=\bigcup_{\lambda \in(0,1]} \lambda(I H) \int_{a}^{b} F_{\lambda}(t) \mathrm{d} t \\
& =\bigcup_{\lambda \in(0,1]} \lambda\left[(H) \int_{a}^{b} F_{\lambda}^{-} \mathrm{d} t,(H) \int_{a}^{b} F_{\lambda}^{+} \mathrm{d} t\right] .
\end{aligned}
$$

For brevity, we write $\tilde{F}(t) \in F H[a, b]$.

Definition 4.4. Let $\tilde{F}:[a, b] \rightarrow \tilde{R}$. If the interval-valued function $\left.F_{\lambda}(t)=\left[F_{\lambda}^{-}(t), F_{\lambda}^{+}(t)\right)\right]$ is AP-Henstock integrable on $[a, b]$ for any $\lambda \in(0,1]$, then $\tilde{F}(t)$ is called AP-Henstock integrable on $[a, b]$ and the integral value is defined by

$$
\begin{aligned}
(A P F H) \int_{a}^{b} \tilde{F}(t) \mathrm{d} t & :=\bigcup_{\lambda \in(0,1]} \lambda(A P I H) \int_{a}^{b} F_{\lambda}(t) \mathrm{d} t \\
& =\bigcup_{\lambda \in(0,1]} \lambda\left[(A P H) \int_{a}^{b} F_{\lambda}^{-} d t,(A P H) \int_{a}^{b} F_{\lambda}^{+} \mathrm{d} t\right] .
\end{aligned}
$$

We write $\tilde{F}(t) \in A P F H[a, b]$.

Theorem 4.1. $\tilde{F} \in A P F H[a, b]$, then $(A P F H) \int_{a}^{b} \tilde{F}(t) \mathrm{d} t \in \tilde{R}$ and

$$
\left((A P F H) \int_{a}^{b} \tilde{F}(t) \mathrm{d} t\right)_{\lambda}=\bigcap_{n=1}^{\infty}(A P I H) \int_{a}^{b} F_{\lambda_{n}}(t) \mathrm{d} t,
$$

where $\lambda_{n}=\left[1-\frac{1}{(n+1)}\right] \lambda$.

Proof. Let $H:(0,1] \rightarrow I_{R}$, be defined by

$$
H(\lambda)=\left[(A P H) \int_{a}^{b} F_{\lambda}^{-}(t) \mathrm{d} t,(A P H) \int_{a}^{b} F_{\lambda}^{+}(t) \mathrm{d} t\right] .
$$

Since $F_{\lambda}^{-}(t)$ and $F_{\lambda}^{+}(t)$ are increasing and decreasing on $\lambda$ respectively, therefore, when $0<\lambda_{1} \leq \lambda_{2} \leq 1$, we have $F_{\lambda_{1}}^{-}(t) \leq F_{\lambda_{2}}^{-}(t), \quad F_{\lambda_{1}}^{+}(t) \geq F_{\lambda_{2}}^{+}(t)$, on $[a, b]$. From Theorem 3.5 we have

$$
\left[(A P H) \int_{a}^{b} F_{\lambda_{1}}^{-}(t) \mathrm{d} t,(A P H) \int_{a}^{b} F_{\lambda_{1}}^{+}(t) \mathrm{d} t\right] \supset\left[(A P H) \int_{a}^{b} F_{\lambda_{2}}^{-}(t) \mathrm{d} t,(A P H) \int_{a}^{b} F_{\lambda_{2}}^{+}(t) \mathrm{d} t\right] .
$$

From Theorem 3.2 and Lemma 4.1 we have

$$
(A P F H) \int_{a}^{b} \tilde{F}(t) \mathrm{d} t:=\bigcup_{\lambda \in(0,1]} \lambda\left[(A P H) \int_{a}^{b} F_{\lambda}^{-} \mathrm{d} t,(A P H) \int_{a}^{b} F_{\lambda}^{+} \mathrm{d} t\right] \in \tilde{R}
$$

and for all $\lambda \in(0,1], \quad\left[(A P F H) \int_{a}^{b} \tilde{F}(t) \mathrm{d} t\right]_{\lambda}=\bigcap_{n=1}^{\infty}(A P I H) \int_{a}^{b} F_{\lambda_{n}}(t) \mathrm{d} t$, where $\lambda_{n}=\left[1-\frac{1}{(n+1)}\right] \lambda$

Theorem 4.2. If $\tilde{F}, \tilde{G} \in A P F H[a, b]$ and $\beta, \gamma \in R$. Then $\beta \tilde{F}+\gamma \tilde{G} \in A P F H[a, b]$ and

$$
(A P F H) \int_{a}^{b}(\beta \tilde{F}+\gamma \tilde{G}) \mathrm{d} t=\beta(A P F H) \int_{a}^{b} \tilde{F}(t) \mathrm{d} t+\gamma(A P F H) \int_{a}^{b} \tilde{G}(t) \mathrm{d} t .
$$

Proof. If $\tilde{F}, \tilde{G} \in A P F H[a, b]$, then the interval-valued function 
$F_{\lambda}(t)=\left[F_{\lambda}^{-}(t), F_{\lambda}^{+}(t)\right]$ and $G_{\lambda}(t)=\left[G_{\lambda}^{-}(t), G_{\lambda}^{+}(t)\right]$ are AP-Henstock integrable on $[a, b]$ for any $\lambda \in(0,1]$ and $(A P F H) \int_{a}^{b} \tilde{F}(t) \mathrm{d} t=\bigcup_{\lambda \in(0,1]} \lambda(A P I H) \int_{a}^{b} F_{\lambda}(t) \mathrm{d} t$ and $(A P F H) \int_{a}^{b} \tilde{G}(t) \mathrm{d} t=\bigcup_{\lambda \in(0,1]} \lambda(A P I H) \int_{a}^{b} G_{\lambda}(t) \mathrm{d} t$. From Theorem 3.3 we have $\beta F_{\lambda}+\gamma G_{\lambda} \in A P I H[a, b]$ and $(A P I H) \int_{a}^{b}\left(\beta F_{\lambda}+\gamma G_{\lambda}\right) \mathrm{d} t=\beta(A P I H) \int_{a}^{b} F_{\lambda} \mathrm{d} t+\gamma(A P I H) \int_{a}^{b} G_{\lambda} \mathrm{d} t$ for any $\lambda \in(0,1]$.

Hence $\beta \tilde{F}+\gamma \tilde{G} \in A P F H[a, b]$ and

$$
\begin{aligned}
(A P F H) \int_{a}^{b}(\beta \tilde{F}+\gamma \tilde{G}) \mathrm{d} t & =\bigcup_{\lambda \in(0,1]} \lambda(A P I H) \int_{a}^{b}\left(\beta F_{\lambda}+\gamma G_{\lambda}\right) \mathrm{d} t \\
& =\bigcup_{\lambda \in(0,1]} \lambda\left(\beta(A P I H) \int_{a}^{b} F_{\lambda} \mathrm{d} t+\gamma(A P I H) \int_{a}^{b} G_{\lambda} \mathrm{d} t\right) \\
& =\beta \bigcup_{\lambda \in(0,1]} \lambda(A P I H) \int_{a}^{b} F_{\lambda} \mathrm{d} t+\gamma \bigcup_{\lambda \in(0,1]} \lambda(A P I H) \int_{a}^{b} G_{\lambda} \mathrm{d} t \\
& =\beta(A P F H) \int_{a}^{b} \tilde{F}(t) \mathrm{d} t+\gamma(A P F H) \int_{a}^{b} \tilde{G}(t) \mathrm{d} t .
\end{aligned}
$$

Theorem 4.3. If $\tilde{F} \in A P F H[a, c]$ and $\tilde{F} \in A P F H[c, b]$, then $\tilde{F} \in A P F H[a, b]$ and

$$
(A P F H) \int_{a}^{b} \tilde{F}(t) \mathrm{d} t=(A P F H) \int_{a}^{c} \tilde{F}(t) \mathrm{d} t+(A P F H) \int_{c}^{b} \tilde{F}(t) \mathrm{d} t .
$$

Proof. If $\tilde{F} \in A P F H[a, c]$ and $\tilde{F} \in A P F H[c, b]$, then the interval-valued function $F_{\lambda}(t)=\left[F_{\lambda}^{-}(t), F_{\lambda}^{+}(t)\right]$ is AP-Henstock integrable on $[a, c]$ and $[c, b]$ for any $\lambda \in(0,1]$ and $(A P F H) \int_{a}^{c} \tilde{F}(t) \mathrm{d} t=\bigcup_{\lambda \in(0,1]} \lambda(A P I H) \int_{a}^{c} F_{\lambda}(t) \mathrm{d} t$ and $(A P F H) \int_{c}^{b} \tilde{F}(t) \mathrm{d} t=\bigcup_{\lambda \in(0,1]} \lambda(A P I H) \int_{c}^{b} F_{\lambda}(t) \mathrm{d} t$. From Theorem 3.4 we have $F_{\lambda} \in A P I H[a, b]$ and $(A P I H) \int_{a}^{b} F_{\lambda} \mathrm{d} t=(A P I H) \int_{a}^{c} F \mathrm{~d} t+(A P I H) \int_{c}^{b} F_{\lambda} \mathrm{d} t$ for any $\lambda \in(0,1]$. Hence $\tilde{F} \in A P F H[a, b]$ and

$$
\begin{aligned}
(A P F H) \int_{a}^{b} \tilde{F}(t) \mathrm{d} t & =\bigcup_{\lambda \in(0,1]} \lambda(A P I H) \int_{a}^{b} F_{\lambda}(t) \mathrm{d} t \\
& =\bigcup_{\lambda \in(0,1]} \lambda\left((A P I H) \int_{a}^{c} F_{\lambda}(t) \mathrm{d} t+(A P I H) \int_{c}^{b} F_{\lambda}(t) \mathrm{d} t\right) \\
& =\bigcup_{\lambda \in(0,1]} \lambda(A P I H) \int_{a}^{c} F_{\lambda}(t) \mathrm{d} t+\bigcup_{\lambda \in(0,1]} \lambda(A P I H) \int_{c}^{b} F_{\lambda}(t) \mathrm{d} t \\
& =(A P F H) \int_{a}^{c} \tilde{F}(t) \mathrm{d} t+(A P F H) \int_{c}^{b} \tilde{F}(t) \mathrm{d} t .
\end{aligned}
$$


Theorem 4.4. If $\tilde{F}(t) \leq \tilde{G}(t)$ nearly everywhere on $[a, b]$ and $\tilde{F}, \tilde{G} \in A P F H[a, b]$, then

$$
(A P F H) \int_{a}^{b} \tilde{F}(t) \mathrm{d} t \leq(A P F H) \int_{a}^{b} \tilde{G}(t) \mathrm{d} t
$$

Proof. If $\tilde{F}(t) \leq \tilde{G}(t)$ nearly everywhere on $[a, b]$ and $\tilde{F}, \tilde{G} \in A P F H[a, b]$, then $F_{\lambda}(t) \leq G_{\lambda}(t)$ nearly everywhere on $[a, b]$ for any $\lambda \in(0,1]$ and $F_{\lambda}(t)$ and $G_{\lambda}(t)$ are AP-Henstock integrable on $[a, b]$ for any $\lambda \in(0,1]$ and $(A P F H) \int_{a}^{b} \tilde{F}(t) \mathrm{d} t=\bigcup_{\lambda \in(0,1]} \lambda(A P I H) \int_{a}^{b} F_{\lambda}(t) \mathrm{d} t$ and $(A P F H) \int_{a}^{b} \tilde{G}(t) \mathrm{d} t=\bigcup_{\lambda \in(0,1]} \lambda(A P I H) \int_{a}^{b} G_{\lambda}(t) \mathrm{d} t$. From Theorem 3.5 we have $(A P I H) \int_{a}^{b} F_{\lambda}(t) \mathrm{d} t \leq(A P I H) \int_{a}^{b} G_{\lambda}(t) \mathrm{d} t$ for any $\lambda \in(0,1]$. Hence

$$
\begin{aligned}
(A P F H) \int_{a}^{b} \tilde{F}(t) \mathrm{d} t & =\bigcup_{\lambda \in(0,1]} \lambda(A P I H) \int_{a}^{b} F_{\lambda}(t) \mathrm{d} t \\
& \leq \bigcup_{\lambda \in(0,1]} \lambda(A P I H) \int_{a}^{b} G_{\lambda}(t) \mathrm{d} t \\
& =(A P F H) \int_{a}^{b} \tilde{G}(t) \mathrm{d} t .
\end{aligned}
$$

\section{Conclusion}

In this paper, we have a tendency to introduce the concept of the AP-Henstock integrals of interval-valued functions and fuzzy number-valued functions and investigate some properties of those integrals.

\section{References}

[1] Wu, C.X. and Gong, Z. (2000) On Henstock Integrals of Interval-Valued Functions and Fuzzy-Valued Functions. Fuzzy Sets and Systems, 115, 377-391. https://doi.org/10.1016/S0165-0114(98)00277-2

[2] Henstock, R. (1963) Theory of Integration. Butterworth, London.

[3] Peng-Yee, L. (1989) Lanzhou Lectures on Henstock Integration. World Scientific, Singapore.

[4] Hamid, M.E. and Elmuiz, E.H. (2016) On Henstock-Stieljes Integrals of Interval-Valued Functions and Fuzzy-Valued Functions. Journal of Applied Mathematics and Physics, 4, 779-786. https://doi.org/10.4236/jamp.2016.44088

[5] Park, J.M., Park, C.G., Kim, J.B., Lee, D.H. and Lee, W.Y. (2004) The Integrals of s-Perron, sap-Perron and ap-McShane. Czechoslovak Mathematical Journal, 54, 545-557. https://doi.org/10.1007/s10587-004-6407-7

[6] Nanda, S. (1989) On Integration of Fuzzy Mappings. Fuzzy Sets and Systems, 32, 95-101. https://doi.org/10.1016/0165-0114(89)90090-0

[7] Wu, C.X. and Ma, M. (1991) Embedding Problem of Fuzzy Number Spaces: Part I. Fuzzy 
Sets and Systems, 44, 33-38. https://doi.org/10.1016/0165-0114(91)90030-T

[8] Wu, C.X. and Ma, M. (1992) Embedding Problem of Fuzzy Number Spaces: Part II. Fuzzy Sets and Systems, 45, 189-202. https://doi.org/10.1016/0165-0114(92)90118-N

[9] Cheng, Z.L. and Demou, W. (1983) Extension of the Integral of Interval-Valued Function and the Integral of Fuzzy-Valued Function. Fuzzy Math, 3, 45-52.

Submit or recommend next manuscript to SCIRP and we will provide best service for you:

Accepting pre-submission inquiries through Email, Facebook, LinkedIn, Twitter, etc. A wide selection of journals (inclusive of 9 subjects, more than 200 journals)

Providing 24-hour high-quality service

User-friendly online submission system

Fair and swift peer-review system

Efficient typesetting and proofreading procedure

Display of the result of downloads and visits, as well as the number of cited articles

Maximum dissemination of your research work

Submit your manuscript at: http://papersubmission.scirp.org/

Or contact am@scirp.org 